\title{
Clinical Features of Patients with Asthma, Bronchiectasis, and Combination of Both Conditions
}

\author{
Fatih Uzer, Zehra Boztepe, Aykut Cilli \\ Department of Respiratory Disease, Akdeniz University Hospital, Antalya, Turkey
}

\section{Abstract}

Background: Asthma and bronchiectasis are critical diseases for public health. In some patients, asthma and bronchiectasis exist together, and bronchiectasis negatively affects asthma control. This study aimed to evaluate the clinical impacts of coexisting asthma and bronchiectasis on patients. Methods: This was a retrospective single-center study on data collected from electronic medical records between January 2014 and January 2017. Patients were divided into three groups, wherein asthma $(n=33)$, bronchiectasis $(n=33)$, and coexisting asthma and bronchiectasis $(n=33)$ were compared. Asthma diagnosis was made according to the guidelines of Global Initiative for Asthma. Confirmation of bronchiectasis diagnosis was made by high-resolution computed tomography. Patients with cystic fibrosis and those who have asthma-chronic obstructive pulmonary disease overlap syndrome were excluded from the study. Results: Medical records of 1467 patients were analyzed. Among them, well-matching 99 patients in terms of gender and comorbid diseases were taken (33 of them had only asthma, 33 had only bronchiectasis, and 33 had coexisting asthma and bronchiectasis). Forty (40.5\%) men and 59 (59.5\%) women with a mean age of $45.0 \pm 5.2$ were included in the study. Asthma and bronchiectasis coexistence had lower forced expiratory volume in $1 \mathrm{~s}(\mathrm{FEV})(\%)$, forced vital capacity $(\mathrm{FVC})(\%), \mathrm{FEV}_{1} / \mathrm{FVC}(\%)$, and $\mathrm{FEV}_{1}(\mathrm{~L})$ ratios $(P=0.0001)$. Moreover, total immunoglobulin E levels were the highest in this group $(P=0.0001)$. The most frequently observed microorganism in the culture tests was Pseudomonas aeruginosa in the bronchiectasis-only as well as in asthma + bronchiectasis groups. Conclusions: Asthmatic patients who have bronchiectasis revealed lower asthma control levels and worse respiratory function test results than other groups.

Keywords: Asthma, bronchiectasis, coexist, control

\section{INTRODUCTION}

Asthma is a heterogeneous disorder involving recurrent episodes of airway obstruction, airway inflammation, and bronchial hyperactivity that affects a considerable portion of the population. Asthma can be triggered by allergens, exposure to irritants, pollutants, and respiratory infections..$^{[1,2]}$

Bronchiectasis is defined as an irreversible and abnormal dilatation of the bronchioles and represents the final stage of various pathological processes. It is typically associated with chronic sputum production with a possibility of bacterial colonization in the lower airways. Inflammation in the airways causes frequent exacerbations, which necessitate hospitalization. $^{[2,3]}$

Asthma and bronchiectasis have different pathophysiologies, diagnostic methods, distinct treatment, and prognosis. Although their physiopathologies are different, they are both

\begin{tabular}{|l|l|}
\hline \multicolumn{3}{|c|}{ Access this article online } \\
\hline Quick Response Code: & Website: \\
\hline & www.ijrc.in \\
\hline & \\
\end{tabular}

classified under obstructive lung diseases. ${ }^{[4]}$ In many patients, asthma and bronchiectasis coexist, and bronchiectasis is shown to worsen asthma control, due to its pulmonary complications. In various studies, it was shown that $3 \%-28 \%$ of asthmatic patients also have bronchiectasis. ${ }^{[5,6]}$ In the presence of coexisting bronchiectasis, most patients are reported as severe asthma. ${ }^{[6]}$

In some studies, it has been argued that bronchiectasis in severe asthma may be an asthma phenotype. ${ }^{[7]}$ In other studies, the presence of bronchiectasis in severe asthma may be treated with anti-immunoglobulin E $(\operatorname{IgE}) \cdot{ }^{[8]}$ Although the coexistence

Address for correspondence: Dr. Fatih Uzer, Department of Chest Diseases, Akdeniz University, Antalya, Turkey. E-mail: fatihuzer@akdeniz.edu.tr

This is an open access journal, and articles are distributed under the terms of the Creative Commons Attribution-NonCommercial-ShareAlike 4.0 License, which allows others to remix, tweak, and build upon the work non-commercially, as long as appropriate credit is given and the new creations are licensed under the identical terms.

For reprints contact: WKHLRPMedknow_reprints@wolterskluwer.com

How to cite this article: Uzer F, Boztepe Z, Cilli A. Clinical features of patients with asthma, bronchiectasis, and combination of both conditions. Indian J Respir Care 2021;10:216-20.

Received: 27-10-2020 Revised: 08-04-2021

Accepted: 09-04-2021 Published: 14-06-2021 
of asthma and bronchiectasis can be regarded as significant, there are very few clinical studies on this subject. Most of the studies are focused on bronchiectasis patients with asthma. Clinical studies with asthma, bronchiectasis, and coexisting asthma and bronchiectasis are very few. The aim of this study is to compare the clinical features of patients with only asthma, only bronchiectasis, and coexisting asthma and bronchiectasis.

\section{Methods}

Patients who visited the Pulmonary Diseases Polyclinic of Akdeniz University Faculty of Medicine between January 1, 2014, and January 1, 2017, were considered for this study. All the information was obtained from the electronic medical records.

Asthma diagnosis was made according to the Global Initiative for Asthma (GINA) guidelines. ${ }^{[1]}$ Asthma diagnosis was confirmed with spirometry, whose respirometry results revealed $12 \%$ and $200-\mathrm{ml}$ change from baseline in forced expiratory volume in $1 \mathrm{~s}\left(\mathrm{FEV}_{1}\right)$ value in patients with wheezing, cough, episodic breathlessness, and chest tightness. In addition, when airway hyperresponsiveness, which is the provocative concentration in a methacholine challenge that causes a $20 \%$ reduction in the $\mathrm{FEV}_{1}$, was below $25 \mathrm{mg} / \mathrm{mL}$, the condition was diagnosed as asthma. High-resolution computed tomography (HRCT) was used for confirming the diagnosis of bronchiectasis. Indications for HRCT included clinical findings compatible with bronchiectasis, such as sputum production, chronic cough, or abnormal chest X-ray results as identified by a radiologist. Based on HRCT findings, patients with bronchiectasis were excluded from pure asthmatics.

Asthma exacerbation was clinically diagnosed by the physician when the patients presented with an increase in the episodes of cough, shortness of breath, wheezing, or chest tightness, or a combination of these symptoms. They were treated with rapid-acting inhaled $\beta$-agonists and steroids.

Bronchiectasis exacerbation is characterized by dyspnea, sputum purulence, and an increase in the sputum production. Sociodemographic information (e.g., age, sex, and history of cigarette smoking) of all the cases, comorbidities, physical examination findings in the lungs (crackles and wheezes), lung function test results (e.g., $\mathrm{FEV}_{1}$, forced vital capacity [FVC], and $\mathrm{FEV}_{1} / \mathrm{FVC}$ ratio), sputum culture results, atopy, and drug use were recorded. Pulmonary function tests were conducted with a spirometer. The spirometer was calibrated according to the operator's manual, once daily with a 3-L syringe. Each patient was tested at least thrice. The best value was expressed as a percentage of the predicted value and as an absolute value. Atopy was assessed with skin prick tests for 11 common allergens (Dermatophagoides farinae, Dermatophagoides pteronyssinus, Cladosporium, Aspergillus mix, dog hair, cat fur, grass, latex, Alternaria, Olea europaea, cereals, positive histamine control, and negative saline control). Skin prick test results were assessed as positive if the weal was at least $3 \mathrm{~mm}$ with no reaction to the negative control. Total $\mathrm{IgE}$ levels were also recorded. Patients with asthma-chronic obstructive pulmonary disease overlap syndrome and those with cystic fibrosis were excluded from the study. None of the asthma patients with bronchiectasis were diagnosed with allergic bronchopulmonary aspergillosis (ABPA). The Ethics Committee of the Akdeniz University approved waiver of consent (approved on March 3, 2018, under number 2018/225).

Statistical analysis was performed with PASW 20 (IBM Corp. Released 2011. IBM SPSS Statistics for Windows, version 20.0. Armonk, NY: IBM Corp.). To define sample frequency distribution, the mean and standard deviation values were used as descriptive statistics. The Kolmogorov-Smirnov test was conducted to determine the normal distribution of continuous variables. The Chi-square significance test was performed for the analysis of categorical variables. The Kruskal-Wallis test was conducted to compare three groups of variables, since continuous variables did not show normal distribution. Statistical significance level was accepted as 0.05 .

\section{ResULTS}

Medical records of 1467 patients were analyzed. $81.9 \%$ (1202) of them had only asthma, 13.5\% (199) of them has only bronchiectasis, and 2.2\% (33) of them had diagnosis of both asthma and bronchiectasis. Among them, well-matching 99 patients in terms of gender and comorbid diseases (hypertension, diabetes mellitus, and coronary artery disease) were taken (33 of them had only asthma, 33 had only bronchiectasis, and 33 had both asthma and bronchiectasis). Forty (40.5\%) men and 59 (59.5\%) women with a mean age of $45.0 \pm 5.2$ years were included in the study. $27.7 \%$ (27) of the patients had a history of smoking. Total IgE levels were found as $320320 \pm 75.5 \mathrm{IU} / \mathrm{mL}$. The general characteristics of the patients are given in Table 1. The sociodemographic characteristics, clinical findings, and pulmonary function tests of the groups are compared in Table 2 . According to this, in coexisting asthma and bronchiectasis, patients had lower $\mathrm{FEV}_{1}(\%), \mathrm{FVC}(\%), \mathrm{FEV}_{1} / \mathrm{FVC}(\%)$, and $\mathrm{FEV}_{1}(\mathrm{~L})$ ratios $(P=0.0001)$. Moreover, total IgE levels were the highest in this group $(P=0.0001)$.

\begin{tabular}{llc}
\hline \multicolumn{3}{l}{ Table 1: General characteristics of } \\
\hline Characteristics & Features & Values \\
\hline Age (Mean \pm SD) & & $45 \pm 5.2$ \\
Sex $n(\%)$ & Female & $59(59.5)$ \\
& Male & $40(40.5)$ \\
Smoking status $n(\%)$ & Ex-smoker & $5(5.1)$ \\
& Smoker & $22(22.2)$ \\
& None & $61(61.6)$ \\
& Unkown & $11(11.1)$ \\
Total immunoglobulin E (mean)/IU/mL & & $320 \pm 75,5$ \\
Number of exacerbations/per year & & 0.99 \\
Number of hositalizations/per year & & 0.36 \\
\hline
\end{tabular}


Uzer, et al:: Clinical features of patients with asthma, bronchiectasis, or both

\begin{tabular}{|c|c|c|c|c|}
\hline & Bronchiectasis ( $n: 33)$ & Bronchiectasis + Asthma ( $n: 33)$ & Asthma ( $n: 33)$ & $P$ \\
\hline Age/year $($ Mean \pm SD) & $45.2 \pm 2.3$ & $45.4 \pm 2.1$ & $45.2 \pm 1.9$ & 0.998 \\
\hline Gender, Male/Female & $13 / 20$ & $13 / 20$ & $14 / 19$ & 0.959 \\
\hline Smoking status & & & & 0.092 \\
\hline Ex-smoker $n(\%)$ & $1(3)$ & $0(0)$ & $4(12.1)$ & \\
\hline Smoker $n(\%)$ & $2(6)$ & $10(30.3)$ & $10(30.3)$ & \\
\hline None $n(\%)$ & $25(75.7)$ & $21(63.6)$ & $15(45.4)$ & \\
\hline Unknown $n(\%)$ & 5 & $2(6)$ & $4(12.1)$ & \\
\hline Total immunoglobulin E (Mean)/IU/mL & $193.6 \pm 112.5$ & $440.4 \pm 114.6$ & $328.0 \pm 64.2$ & 0.0001 \\
\hline Number of exacerbations/per year & $1.09(0-6)$ & $1.21(0-8)$ & $0.42(0-2)$ & 0.056 \\
\hline Number of hospitalizations/per year & $0.36(0-4)$ & $0.64(0-8)$ & $0.09(0-1)$ & 0.210 \\
\hline $\mathrm{FEV}_{1}(\%)$ & $56.52 \pm 3.45$ & $50.98 \pm 4.28$ & $83.82 \pm 3.04$ & 0.0001 \\
\hline FVC $(\%)$ & $60.46 \pm 2.94$ & $60.9 \pm 4.14$ & $86.2 \pm 2.51$ & 0.0001 \\
\hline $\mathrm{FEV}_{1} / \mathrm{FVC}(\%)$ & $76.42 \pm 2.46$ & $66.77 \pm 2.31$ & $80.61 \pm 1.83$ & 0.0001 \\
\hline $\mathrm{FEV}_{1}(\mathrm{~L})$ & $1.61 \pm 0.11$ & $1.45 \pm 0.12$ & $2.45 \pm 0.15$ & 0.0001 \\
\hline FVC (L) & $2.08 \pm 0.13$ & $2.1 \pm 0.16$ & $3.05 \pm 0.19$ & 0.0001 \\
\hline
\end{tabular}

Table 3: Asthma classification according to GINA of patients

\begin{tabular}{lccc}
\hline & $\begin{array}{c}\text { Bronchiectasis } \\
+ \text { Asthma } \boldsymbol{n}(\%)\end{array}$ & $\begin{array}{c}\text { Asthma } \\
\boldsymbol{n}(\%)\end{array}$ & $\boldsymbol{P}$ \\
\hline Uncontrolled & $12(36.4)$ & $5(15.2)$ & 0.0001 \\
Partly controlled & $5(15.2)$ & $7(21.2)$ & \\
Controlled & $5(15.2)$ & $21(63.6)$ & \\
Unknown & $11(33.3)$ & $0(0)$ & \\
Total & $33(100)$ & $33(100)$ & \\
\hline
\end{tabular}

According to the GINA guidelines, asthma control levels were significantly worse in the patients who have both asthma and bronchiectasis [Table 3]. Moreover, no statistically significant difference was observed between hospitalization rates $(P=0.190)$ among the three groups. The most frequent symptom in the asthma + bronchiectasis group was dyspnea, whereas that in the bronchiectasis group was sputum production. The most frequently observed microorganism in the culture tests was Pseudomonas aeruginosa in the bronchiectasis-only as well as in asthma + bronchiectasis groups [Table 4]. The most commonly used drug in all the groups was identified as the combination of a long-acting beta 2-agonist (LABA) and inhaled corticosteroid (ICS) (LABA + ICS). ICS was the most commonly used drug in the asthma-only group.

\section{DISCUSSION}

Asthma is one of the most common chronic lung diseases and is accompanied by many comorbidities. In a study conducted in Scotland, asthmatic adults were five times more likely to have bronchiectasis than healthy adults. ${ }^{[9]} \mathrm{A}$ few studies examining the association between asthma and bronchiectasis have reported that this association may indicate poor prognosis. ${ }^{[3,6,10,11]}$ Our study revealed no significant difference in hospitalization and exacerbation numbers between the asthma + bronchiectasis group and the asthma-only group, but pulmonary function and asthma control test results were significantly worse in the former group.

A study conducted by Säynäjäkangas et al.$^{[12]}$ reported that asthma was common in the patients hospitalized for bronchiectasis, stating that this was a result of the disease. A South Korean study reported a $2.2 \%$ incidence of bronchiectasis in asthmatic patients, whereas this ratio was reported to be $3 \%$ in a study from Turkey ${ }^{[3,6]}$ Bisaccioni et al. ${ }^{[13]}$ and Padilla-Galo et al ${ }^{[5]}$ found that $24.8 \%$ and $28 \%$ of patients with asthma had accompanying bronchiectasis, respectively. In our study, this ratio was $2.6 \%$. The reason for such differences among studies may be that HRCT control was not performed in all the patients screened for each study. In asthmatic patients, the physician performs HRCT in cases of suspected bronchiectasis. Failure to perform HRCT in subclinical asthmatic cases might be a cause of the low prevalence.

The hypothesis that atopy and asthma have a role in the pathogenesis of bronchiectasis was first suggested in 1939..$^{[14]}$ Since then, many studies have reported a positive relationship between bronchiectasis and atopy; however, some studies did not find any relationship between bronchiectasis and atopy. ${ }^{[6,15,16]}$ In the present study, the $\operatorname{IgE}$ level was found to be statistically significantly higher in the bronchiectasis + asthma group compared to the other groups. High serum IgE level was found in patients with bronchiectasis but without ABPA, suggesting that an elevated IgE level may be associated with increased susceptibility to bronchial infections, which in turn result in bronchiectasis. In a study conducted by Luján et al..$^{[17]}$ on the prevalence of bronchiectasis in asthmatic patients using oral steroids, no statistically significant difference was detected in immunoglobulin levels, except for the immunoglobulin $\mathrm{M}(\operatorname{IgM})$ levels. The authors attributed this finding to possible 
Uzer, et al.: Clinical features of patients with asthma, bronchiectasis, or both

\begin{tabular}{lcc}
\hline Table 4: Sputum culture and obtained pathogens results of patients $(\boldsymbol{n})$ & \\
\hline & Bronchiectasis & Bronchiectasis + Asthma \\
\hline Acinetobacter baumannii & 0 & 2 \\
Candida albicans & 1 & 3 \\
Candida tropcalis & 0 & 1 \\
E. coli & 0 & 3 \\
Haemophilus influenzae & 8 & $0.028^{*}$ \\
Haemophilus parahaemolyticus & 1 & 0 \\
Klebsiella oxytoca & 1 & 0 \\
Moraxella catarrhalis & 1 & 8 \\
Pseudomonas aeruginosa & 11 & 1 \\
Staphylococcus aureus & 0 & 0 \\
Staphylococcus epidermidis & 1 & 1 \\
Streptococcus pneumoniae & 0 & 0 \\
None & 8 & 7 \\
Unknown & 21 &
\end{tabular}

near-term infections in bronchiectasis patients. In their study, IgE was not detected, indicating the allergy burden of the patient, and the immunoglobulins examined were immunoglobulin A, IgG, and IgM.

Oguzulgen et al..$^{[6]}$ found that $49 \%$ of asthmatic patients who had bronchiectasis had severe persistent asthma, whereas in a study by Bisaccioni et al., this rate was $94.6 \%{ }^{[13]}$ In the present study, $38 \%$ of asthmatic patients with bronchiectasis had uncontrolled asthma. Further, asthma was significantly better controlled in the asthma-only group as compared to the other groups.

A previous study reported that bronchiectasis patients with asthma have a 2.6-fold higher rate of exacerbation than nonasthmatic bronchiectasis patients. ${ }^{[10]}$ The authors attributed this to an increased risk of possible pneumonia in asthmatic patients using ICS. Although the use of ICS in our study was higher in the asthma-only group, no statistically significant difference was found in the numbers of exacerbations and hospitalizations between the asthma + bronchiectasis group and the bronchiectasis-only group.

The diagnosis of asthma is based on a compatible clinical history and characteristic findings from a series of pulmonary function tests. Most of the $\mathrm{FEV}_{1}$ and $\mathrm{FEV}_{1} / \mathrm{FVC}$ parameters are used in the evaluation of the airway obstruction. ${ }^{[1,2]} \mathrm{In}$ bronchiectasis, obstructive impairment is the most frequent finding, but a very low FVC can also be observed in the advanced stage of the disease, which presents with extensive lung damage. ${ }^{[18,19]}$ In the present study, when the pulmonary function tests of all the three groups were compared, the asthma + bronchiectasis group exhibited significantly lower levels for all parameters. This finding suggests that the patients are functionally more affected if both the conditions coexist.

The role of bacteria in diagnosis and exacerbation of bronchiectasis is well defined; however, the role of pathogenic bacteria in the lower airways of patients with asthma is unclear. Bacteria, through the activation of the innate immune response such as the toll-like receptors, may induce the release of inflammatory cytokines such as interleukin-8 and tumor necrosis factor- $\alpha$ that could induce neutrophilic inflammation. One of the most commonly isolated microorganisms in sputum of patients with bronchiectasis is $P$. aeruginosa, but it is not very common in the sputum of asthmatic patients. In our study, $P$. aeruginosa was the most commonly isolated microorganism both in the bronchiectasis group and in the bronchiectasis + asthma group. The interaction between $P$. aeruginosa and the bronchial epithelium was studied in bronchial mucosal probes cultured in tissue culture medium. It was possible to demonstrate that, even after loss of the mucus layer, adherence between the bacteria and the bronchial epithelium does not take place if ciliary function remains intact. $^{[20,21]}$ This may lead to chronic lung infection with P. aeruginosa.

The most important limitation of our study is its retrospective design, which may have led to the selection bias. A prospective screening trial with HRCT in newly diagnosed asthmatic patients might provide more reliable results. However, the HRCT screening of all the asthmatic patients is not ethical or cost-effective.

\section{Conclusions}

Our study established that the asthmatic patients with bronchiectasis exhibited worse respiratory function test results than the other groups, whereas no significant difference was observed between the groups in terms of hospitalizations and exacerbations. Extensively planned prospective research is necessary in this regard.

\section{Financial support and sponsorship Nil.}

\section{Conflicts of interest}

There are no conflicts of interest. 


\section{RefEREnCES}

1. Global Initiative for Asthma (GINA). Global Strategy for Asthma Management and Prevention; 2018. Available from: https://ginasthma. org/2018-gina-report-global-strategy-for-asthma-management-andprevention/. [Last accessed on 2018 Oct 10].

2. Whitters D, Stockley R. Immunity and bacterial colonisation in bronchiectasis. Thorax 2012;67:1006-13.

3. Kang HR, Choi GS, Park SJ, Song YK, Kim JM, Ha J, et al. The effects of bronchiectasis on asthma exacerbation. Tuberc Respir Dis (Seoul) 2014;77:209-14

4. Mysliwiec V, Pina JS. Bronchiectasis: The 'other' obstructive lung disease. Postgrad Med 1999;106:123-6, 128-31.

5. Padilla-Galo A, Olveira C, Fernández de Rota-Garcia L, Marco-Galve I, Plata AJ, Alvarez A, et al. Factors associated with bronchiectasis in patients with uncontrolled asthma; the NOPES score: A study in 398 patients. Respir Res 2018;19:43.

6. Oguzulgen IK, Kervan F, Ozis T, Turktas H. The impact of bronchiectasis in clinical presentation of asthma. South Med J 2007;100:468-71.

7. Perez-Miranda J, Traversi L, Polverino E. Bronchiectasis in severe asthma: A distinct phenotype? Curr Opin Pulm Med Ocak 2019;25:71-8.

8. Carpagnano GE, Scioscia G, Lacedonia D, Curradi G, Foschino Barbaro MP. Severe uncontrolled asthma with bronchiectasis: A pilot study of an emerging phenotype that responds to mepolizumab. J Asthma Allergy 2019;12:83-90.

9. Weatherburn CJ, Guthrie B, Mercer SW, Morales DR. Comorbidities in adults with asthma: Population-based cross-sectional analysis of 1.4 million adults in Scotland. Clin Exp Allergy 2017;47:1246-52.

10. Mao B, Yang JW, Lu HW, Xu JF. Asthma and bronchiectasis exacerbation. Eur Respir J 2016;47:1680-6.

11. Dimakou K, Gousiou A, Toumbis M, Kaponi M, Chrysikos S, Thanos L, et al. Investigation of bronchiectasis in severe uncontrolled asthma. Clin Respir J 2018;12:1212-8.
12. Säynäjäkangas $O$, Keistinen $T$, Tuuponen $T$, Kivelä SL. Links between hospital diagnoses of bronchiectasis and asthma. Allergy 1997;52:1120-2.

13. Bisaccioni C, Aun MV, Cajuela E, Kalil J, Agondi RC, Giavina-Bianchi P. Comorbidities in severe asthma: Frequency of rhinitis, nasal polyposis, gastroesophageal reflux disease, vocal cord dysfunction and bronchiectasis. Clinics (Sao Paulo) 2009;64:769-73.

14. Watson SH, Kibler CS. The role of allergy in bronchiectasis. J Allergy 1939;10:364-76.

15. Murphy MB, Reen DJ, Fitzgerald MX. Atopy, immunological changes, and respiratory function in bronchiectasis. Thorax 1984;39:179-84.

16. Pang J, Chan HS, Sung JY. Prevalence of asthma, atopy, and bronchial hyperreactivity in bronchiectasis: A controlled study. Thorax 1989;44:948-51.

17. Luján M, Gallardo X, Amengual MJ, Bosque M, Mirapeix RM, Domingo C. Prevalence of bronchiectasis in asthma according to oral steroid requirement: Influence of immunoglobulin levels. Biomed Res Int 2013;2013:109219.

18. Lopes AJ, Camilo GB, de Menezes SL, Guimarães FS. Impact of different etiologies of bronchiectasis on the pulmonary function tests. Clin Med Res 2015;13:12-9.

19. Roberts HR, Wells AU, Milne DG, Rubens MB, Kolbe J, Cole PJ, et al. Airflow obstruction in bronchiectasis: Correlation between computed tomography features and pulmonary function tests. Thorax 2000;55:198-204.

20. Philippon S, Streckert HJ, Morgenroth K. In vitro study of the bronchial mucosa during Pseudomonas aeruginosa infection. Virchows Arch A Pathol Anat Histopathol 1993;423:39-43.

21. Ciofu O. Pseudomonas aeruginosa chromosomal beta-lactamase in patients with cystic fibrosis and chronic lung infection. Mechanism of antibiotic resistance and target of the humoral immune response. APMIS Suppl 2003;116:1-47. 